\title{
Upgrading the field-imaging far- infrared line spectrometer for the Stratospheric Observatory for Infrared Astronomy (SOFIA) with KIDs: enabling large sample (extragalactic) surveys
}

Colditz, Sebastian, Looney, Leslie, Bigiel, Frank, Fischer, Christian, Fischer, Jacqueline, et al.

Sebastian Colditz, Leslie W. Looney, Frank Bigiel, Christian Fischer, Jacqueline Fischer, Steven Hailey-Dunsheath, Rodrigo Herrera-Camus, Alfred Krabbe, Henry LeDuc, Tony Wong, Jonas Zmuidzinas, "Upgrading the fieldimaging far-infrared line spectrometer for the Stratospheric Observatory for Infrared Astronomy (SOFIA) with KIDs: enabling large sample (extragalactic) surveys," Proc. SPIE 11453, Millimeter, Submillimeter, and Far-Infrared Detectors and Instrumentation for Astronomy X, 1145334 (13 December 2020); doi: $10.1117 / 12.2560120$ 


\title{
Upgrading the field-imaging far-infrared line spectrometer for the Stratospheric Observatory for Infrared Astronomy (SOFIA) with KIDs: Enabling large sample (extragalactic) surveys
}

Sebastian Colditz*a ${ }^{* a}$ Leslie W. Looney ${ }^{\mathrm{b}}$, Frank Bigiel ${ }^{\mathrm{c}}$, Christian Fischer ${ }^{\mathrm{a}}$, Jacqueline Fischer ${ }^{\mathrm{d}}$, Steven Hailey-Dunsheath ${ }^{\mathrm{e}}$, Rodrigo Herrera-Camus ${ }^{\mathrm{f}}$, Alfred Krabbe ${ }^{\mathrm{a}}$, Henry LeDuc ${ }^{\mathrm{g}}$, Tony Wong ${ }^{\mathrm{b}}$, Jonas Zmuidzinas ${ }^{\mathrm{e}}$

aUniversität Stuttgart, Deutsches SOFIA Institut, Pfaffenwaldring 29, 70569 Stuttgart, Germany;

${ }^{b}$ Department of Astronomy, University of Illinois, 1002 W Green St., Urbana, IL, 61801, USA; ${ }^{c}$ Argelander-Institut für Astronomie, Universität Bonn, Auf dem Hügel 71, 53121 Bonn, Germany; ${ }^{\mathrm{d}}$ George Mason University, Department of Physics \& Astronomy, MS 3F3, 4400 University Drive, Fairfax, VA 22030, USA; ${ }^{\circ}$ California Institute of Technology, 1200 E. California Blvd, Mail Code 301-17, Pasadena, 91125, USA; fDepartamento de Astronomía, Universidad de Concepción, Barrio Universitario, Concepción, Chile; ${ }^{\mathrm{g} J e t}$ Propulsion Laboratory, California Institute of Technology, Pasadena, California 91125, USA

\begin{abstract}
We present the initial design, performance improvements and science opportunities for an upgrade to the Field-Imaging Far-Infrared Line Spectrometer (FIFI-LS).

FIFI-LS efficiently measures fine structure cooling lines, delivering critical constraints of the interstellar medium and starforming environments. SOFIA provides the only FIR observational capability in the world, making FIFI-LS a workhorse for FIR lines, combining optimal spectral resolution and a wide velocity range. Its continuous coverage from 51-203 microns makes FIFI-LS a versatile tool to investigate a multitude of diagnostic lines within our galaxy and in extragalactic environments.

The sensitivity and field-of-view (FOV) of FIFI-LS are limited by its 90s-era photoconductor arrays. These limits can be overcome by upgrading the instrument using the latest developments in Kinetic Inductance Detectors (KIDs). KIDs provide sensitivity gains in excess of 1.4 and allow larger arrays, enabling an increase in pixel count by an order of magnitude. This increase allows a wider FOV and instantaneous velocity coverage. The upgrade provides gains in point source observation speed by a factor $>2$ and in mapping speed by a factor $>3.5$, enabled by the improved sensitivity and pixel count.
\end{abstract}

This upgrade has been proposed to NASA in response to the 2018 SOFIA Next Generation Instrumentation call.

Keywords: Integral Field Spectroscopy, Kinetic Inductance Detectors, Far-Infrared, FIFI-LS, SOFIA

\section{INTRODUCTION}

The Field-Imaging Far-Infrared Line Spectrometer (FIFI-LS $)^{1}$ is the sensitive medium resolution (R 1500) spectrometer for the Stratospheric Observatory for Infrared Astronomy (SOFIA) ${ }^{2}$. FIFI-LS operates two spectral channels from 51-125 $\mu \mathrm{m}$ and 115-203 $\mu \mathrm{m}$ simultaneously. It allows fast mapping of the fine structure cooling lines of the interstellar medium and in star forming environments within and outside of our galaxy. Its wide instantaneous velocity coverage also allows sensitive observations of more distant galaxies that are just resolved by SOFIA's $2.5 \mathrm{~m}$ class telescope. The airborne observatory SOFIA provides routine access to its instrument suite, allowing for servicing, exchange and upgrade of the instruments. This is a major advantage in comparison to space observatories, which usually cannot be upgraded.

*colditz@dsi.uni-stuttgart.de

Millimeter, Submillimeter, and Far-Infrared Detectors and Instrumentation for Astronomy X, edited by Jonas Zmuidzinas, Jian-Rong Gao, Proc. of SPIE Vol. 11453, 1145334

(C) 2020 SPIE · CCC code: 0277-786X/20/\$21 · doi: 10.1117/12.2560120 
The upgrade of FIFI-LS to FIFI+LS presented in this paper is designed to make the best use of this advantage to improve the observatories scientific capabilities and efficiency. Based on the development of new kinetic inductance detector (KID) arrays, the upgrade will enable point source sensitivity gains of a factor of at least 1.4 (potentially up to 2.5) and gains in mapping speed by a factor of at least 3.5 (potentially up to 12). These gains will enable SOFIA observations that would otherwise be infeasible due to the amount of observing time necessary. We have proposed the upgrade presented in this paper including a legacy science program to NASA, in response to the SOFIA Next Generation Instrumentation call 2018. The response of the review board relayed to us was generally favorable; however to date NASA has not made a decision. In Section 2 of this paper, we give an overview of the solid basis of our upgrade concept: the FIFI-LS instrument as it is currently operated on SOFIA. We present the anticipated performance improvements and the details of the upgrade concept in Section 3. In Section 4, we show how the upgrade would open new scientific opportunities.

\section{FIFI-LS: THE SOLID BASIS}

\subsection{FIFI-LS overview}

In-depth information on FIFI-LS was published in Fischer et al. ${ }^{1}$ and Looney et al. ${ }^{3}$. Here we give a coarse overview of the instrument design, concentrating on subsystems and concepts that are relevant to the instrument upgrade.

FIFI-LS is a dual channel imaging spectrometer. Both spectral channels operate simultaneously, allowing observations of two spectral lines at the same time. The long wavelength (red) channel operates between $115 \mu \mathrm{m}$ and $203 \mu \mathrm{m}$ and the short wavelength (blue) channel between $51 \mu \mathrm{m}$ and $125 \mu \mathrm{m}$. The instantaneous spectral coverage is $800-2500 \mathrm{~km} / \mathrm{s}$ in red and $1000-3000 \mathrm{~km} / \mathrm{s}$ in the blue channel, depending on the observed wavelength. The spectral resolution is also wavelength dependent and ranges from $150 \mathrm{~km} / \mathrm{s}$ to $600 \mathrm{~km} / \mathrm{s}$. Spatially, each channel has a 5 by 5 spatial pixel (spaxel) field of view (FOV). In the red channel, the spaxel size on the sky corresponds to 12.2 by 12.5 square arc seconds, in the blue channel to 6.14 by 6.25 square arc seconds.

Both channels are equipped with a reflective integral field unit (IFU) which reorganizes the square $5 \times 5$ spaxel FOV into a $(25+4) \times 1$ spaxel slit, which is coupled into a quasi Littrow mounted spectrometer. Figure 1 shows the IFU and a schematic of how the 2-dimensional FOV is reorganized into a 1-dimensional slit. The IFU comprises three sets of five mirrors each. The first set are the slicer mirrors. These mirrors are in a focal plane of the instrument. They slice the $5 \times 5$ spaxel FOV into five image slices and reimage the entrance pupil for each slice onto one of the five capture mirrors, which form the second set of mirrors. The capture mirrors are positioned in the plane of a pupil image. Each of the five mirrors refocuses its slice onto a slit mirror in the third set of mirrors of the IFU. Therefore, the slit mirrors are again in a focal plane. The focused image of the slit has a length of $25+4$ spaxels at this position. The space for the four additional spaxels are gaps between the five individual slices. These gaps are introduced to avoid crosstalk between the edges of the slices. Finally, the slit mirrors ensure that the pupil image of each slice is correctly centered on the grating once it passes the collimating optics. The dispersing element in each spectrometer channel is a reflective grating. Each grating may be rotated within a total range of about $40^{\circ}$ at sub-arc second precision, to select the observed wavelength. The gratings have been optimized for their specific wavelength ranges. The simulated grating efficiencies are shown in Figure 2. Especially the blue channel grating is highly polarizing towards the ends of the covered wavelengths. The polarization has to be taken into account when optimizing the new detector arrays, when pixels have polarization dependent quantum efficiency (QE).

The core of the instrument are its two 400-pixel Gallium-doped Germanium photoconductor detector arrays ${ }^{4}$. Each array is assembled from 25 modules - one for each spaxel - with 16 pixels in the spectral direction. For the red channel, the modules have been specially designed to apply strong uniform stress to the 16 pixels in order to shift their spectral response towards longer wavelengths. The modules in the blue channel apply only enough stress to ensure the mechanical stability of the pixel stack. The detectors have been assembled pixel by pixel, manually. The pixel yield is $>95 \%$. The photon dominated NEP of the detectors is about $1.5 \times 10^{-16} \mathrm{~W} \mathrm{~Hz}^{-1 / 2}$. The dark NEP is about $5 \times 10^{-18} \mathrm{~W} \mathrm{~Hz}^{-1 / 2}$. The QE as inferred from measurements assuming background limited performance ranges from $25 \%$ to $35 \%{ }^{3}$. Light is coupled to the individual pixels via funnel shaped light cones. The area of a pixel in the focal plane of the detector is about $3.9 \times 3.9 \mathrm{~mm}^{2}$. Each detector module is read out by a Cold Read-out Electronic circuit (CRE). The CREs are specially designed CMOS circuits, originally developed for HERSCHEL-PACS ${ }^{5}$. The detector current is read out by a capacitive feedback, integrating amplifier. A sample-and-hold circuit within the CRE acts as analog memory between integrator and multiplexing circuit. The capacitor voltages are sampled with a rate of $250 \mathrm{~Hz}$. The measurement is done in a sampling up the ramp technique, typically resetting the capacitor voltage every 32 samples. Therefore, the detector electronics supply a rate of about 7.8 ramps per second with 32 samples on each ramp. 


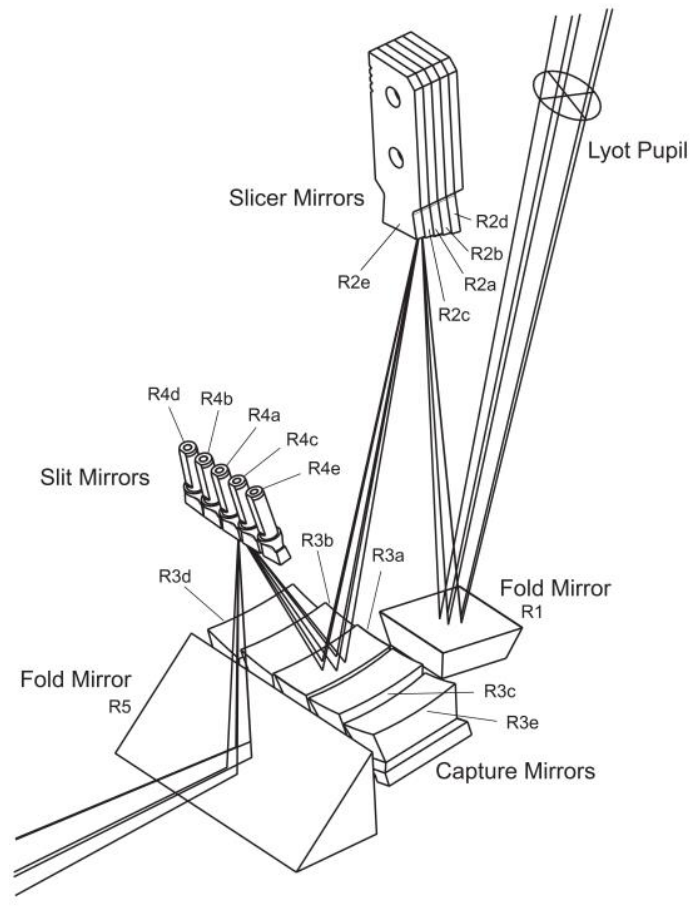

$5 \times 5$ Spaxel FOV at Focal Plane on Slicer Mirrors

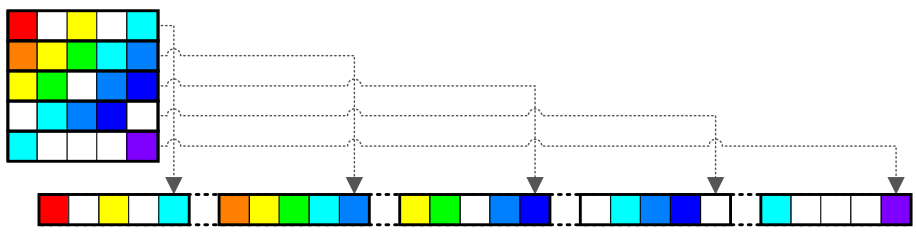

25(+4)x1 Spaxel pseudo Slit at Focal Plane on Slit Mirrors



Figure 1: IFU concept of FIFI-LS. Left ${ }^{3}$ : Close-up view of the IFU mirror components for the red channel, with the rays for the central slice shown. The blue channel is similar. Right: Illustration of the field-imaging concept in FIFI-LS. The 5x5 Spaxel FOV imaged in the plane of the slicer mirrors is sliced into five slices and reorganized on a $(25+4) x 1$-spaxel pseudo slit, imaged in the plane of the slit mirrors. The pseudo slit is dispersed in spectral direction in the spectrometer optics and imaged onto the 400-pixel detector, with 25 pixels in spatial direction along the slit and 16 pixels in spectral direction perpendicular to the slit. The color-coding is an example to display the effects in spectral direction - white spaxels represent a continuous spectrum, colored spaxels a monochromatic spectrum.

Both detectors are cooled to temperatures of $1.6 \mathrm{~K}$ to attain the NEP mentioned above. The spectrometer optics are cooled to $6 \mathrm{~K}$ and the entrance optics to $77 \mathrm{~K}$, reducing the thermal background within the instrument. Cooling of the instrument is accomplished by the four-layer bath cryostat design shown in Figure 3. The first layer is the evacuated cryostat shell at room temperature. The second layer is cooled to $70 \mathrm{~K}$ by a 25 -liter liquid nitrogen vessel attached to a radiation shield and the optical bench for the entrance optics. On the third layer, the cooling is provided by a 35 -liter liquid helium vessel. The optical bench with the IFU and spectrometer optics belongs to this level. Due to the heat influx and the heat produced on the optical bench by electronic components, the temperature is typically $6 \mathrm{~K}$, which is slightly higher than the boiling temperature of liquid helium at $4 \mathrm{~K}$. The innermost layer is occupied by the two 400 pixel detector arrays and the CREs. This layer is cooled by a small 2.8-liter superfluid liquid helium vessel. This vessel is pumped in order to attain the operating temperature of $1.6 \mathrm{~K}$. 


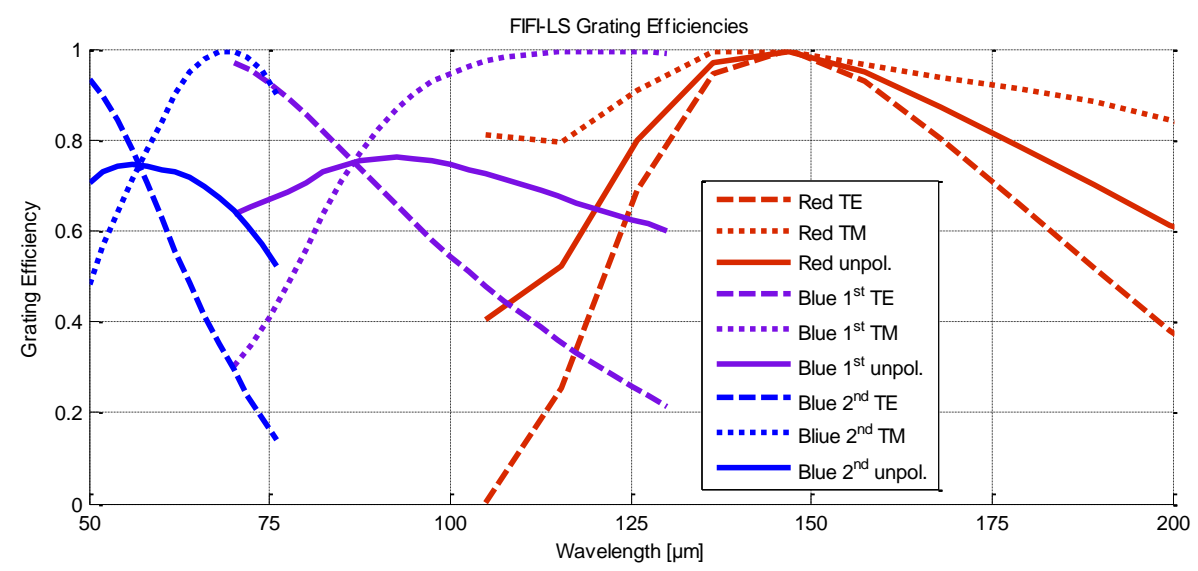

Figure 2: Simulated grating efficiencies of the FIFI-LS gratings. The red channel operates in first order only, for the blue channel first and second order are used - in operation a filter changer switches between filter sets optimized for either order. The gratings have polarizing effects. The TE polarization has the E-field parallel to the grating grooves, while the TM polarization has the E-field perpendicular to the groves. Simulation by Norbert Geis, Max Planck Institute for Extraterrestrial Physics.

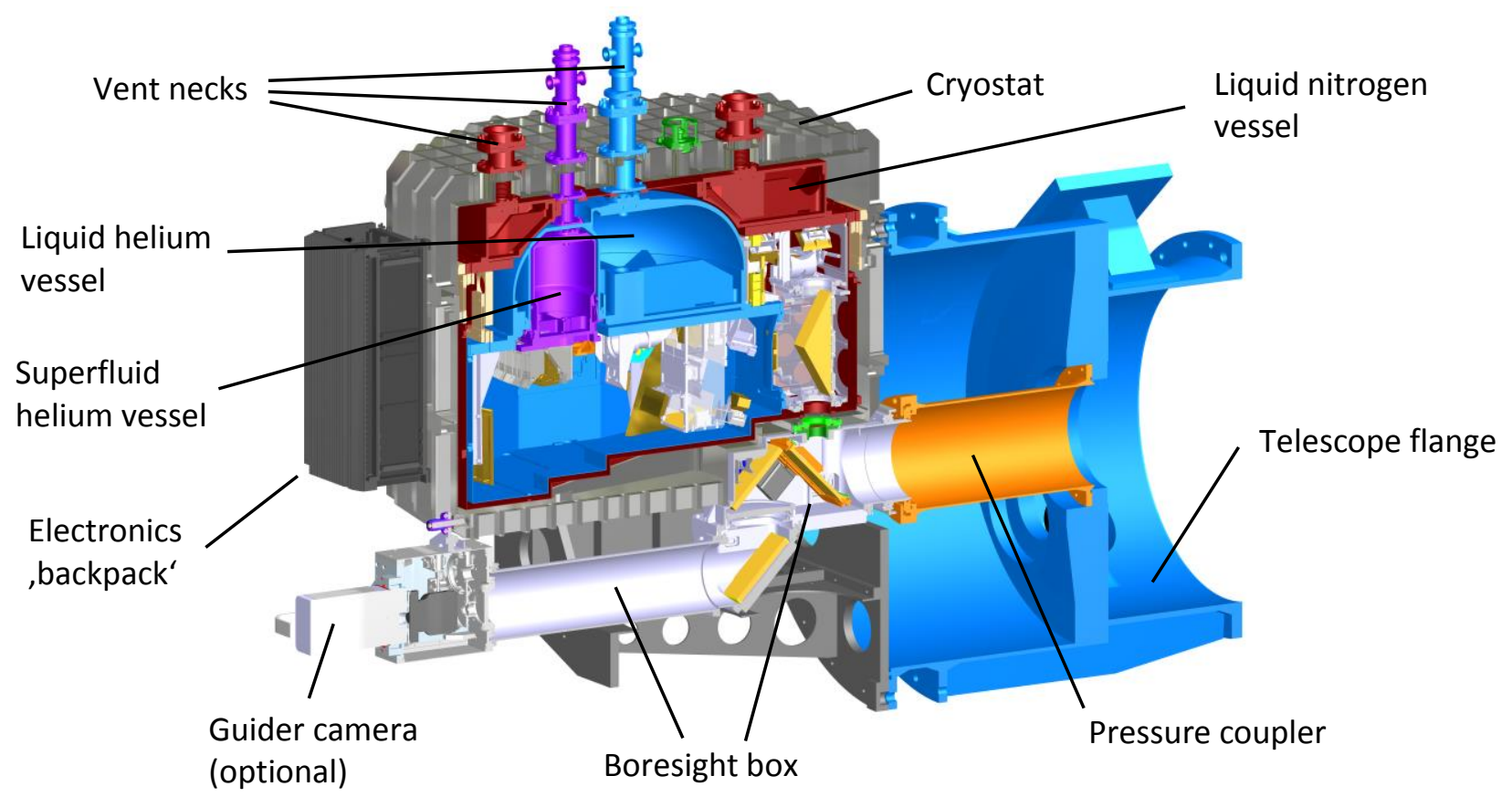

Figure 3: CAD drawing of the FIFI-LS cryostat attached to the SOFIA telescope flange. ${ }^{1}$

\subsection{Science with FIFI-LS}

With its sensitivity, wavelength coverage, spectral and spatial resolution, FIFI-LS plays a major role in investigations of the interstellar medium both extragalactic and within our galaxy, as well as for investigations of (primarily massive) star formation since 2014. The wide wavelength coverage of FIFI-LS allows the observation of a multitude of diagnostic lines in the far infrared (FIR). Table 1 lists all lines observed to date. The key features observed most often with FIFI-LS are the bright FIR fine structure lines of [CII] at $158 \mu \mathrm{m},[\mathrm{OI}]$ at 63 and $146 \mu \mathrm{m},[\mathrm{OIII}]$ at 52 and $88 \mu \mathrm{m},[\mathrm{NII}]$ at $122 \mu \mathrm{m}$, 
[NIII] at $57 \mu \mathrm{m}$, and molecular lines like the $\mathrm{CO}$ rotational lines from $\mathrm{J}=13-12$ up to $\mathrm{J}=38-37$. FIFI-LS observations also provide the underlying continuum flux for sufficiently bright objects. Below we summarize a few of the published FIFI-LS results.

Table 1. Spectral lines observed with FIFI-LS ${ }^{1}$

\begin{tabular}{cc|cc|cc|cc|cc} 
line & $\lambda[\mu \mathrm{m}]$ & line & $\lambda[\mu \mathrm{m}]$ & line & $\lambda[\mu \mathrm{m}]$ & line & $\lambda[\mu \mathrm{m}]$ & line & $\lambda[\mu \mathrm{m}]$ \\
\hline$[\mathrm{OIII}]$ & 51.8 & $\mathrm{CO}$ & 70.9 & $\mathrm{CH} 4$ & 87.3 & $\mathrm{CO}$ & 124.2 & $\mathrm{OH}$ & 163.3 \\
$\mathrm{OH}$ & 55.9 & $\mathrm{CO}$ & 77.1 & {$[\mathrm{OIII}]$} & 88.4 & $\mathrm{CO}$ & 130.4 & $\mathrm{HCN}$ & 169.4 \\
{$[\mathrm{NIII}]$} & 57.3 & $\mathrm{CH}_{4}$ & 80.1 & $\mathrm{OH}$ & 96.3 & {$[\mathrm{OI}]$} & 145.5 & $\mathrm{CO}$ & 174 \\
{$[\mathrm{OI}]$} & 63.2 & $\mathrm{CO}$ & 84.4 & $\mathrm{CO}$ & 96.8 & $\mathrm{CO}_{2}(\mathrm{ice})$ & 146 & $\mathrm{CO}$ & 186 \\
$\mathrm{C}_{2} \mathrm{H}_{2}$ & 68.6 & $\mathrm{OH}$ & 84.5 & $\mathrm{CO}$ & 104.4 & $\mathrm{CO}$ & 153.3 & $\mathrm{CO}$ & 200.3 \\
$\mathrm{CO}$ & 69.1 & $\mathrm{CO}_{2}(\mathrm{ice})$ & 86 & $\mathrm{CO}$ & 118.6 & {$[\mathrm{CII}]$} & 157.7 & & \\
$\mathrm{C}_{2} \mathrm{H}_{2}$ & 69.7 & $\mathrm{CO}$ & 87.2 & {$[\mathrm{NII}]$} & 122 & $\mathrm{CO}$ & 162.8 &
\end{tabular}

FIFI-LS has efficiently mapped complete galaxies in [CII], to study how star formation rates and [CII] emission correlate in different environmental conditions.

For example, Pineda et al. ${ }^{6}$ probed the relationship between SFR and [CII] over a wide range of conditions by mapping the whole disk of the M51 galaxy including its companion M51b. The preliminary results are that SFR and [CII] emission are well correlated over the disk of M51 including the Center, Spiral Arms, and Inter-Arm regions, in good agreement with the KINGFISH galaxy relationship ${ }^{7}$. However, the M51b galaxy shows a significant deficit of [CII] emission with respect to the SFR rate estimated from FIR-continuum. M51b is a barred lenticular galaxy in a post-starburst phase in which massive star-formation is suppressed ${ }^{8}$. The lack of massive star formation in M51b is consistent with the faint [CII] emission detected, but it is inconsistent with the large SFR estimated in this galaxy. The bright $24 \mu \mathrm{m}$ dust continuum emission, which dominates the SFR estimate, is likely heated by an active galactic nucleus, therefore not tracing star formation activity. The observed [CII] deficit in M51b suggests that this galaxy is a nearby analog of ultra-luminous infrared galaxies and represents an important laboratory in which to study the origin of the [CII] deficit observed in these galaxies.

Bigiel et al ${ }^{63}$ presented the results for another full-galaxy [CII] map with FIFI-LS of the nearby spiral galaxy NGC6946. The data allowed disentangling the [CII] luminosities of the arms $(73 \%)$, center $(19 \%)$, and interarm regions $(8 \%)$ of NGC6946. A comparison of the radial profiles of [CII] emission to various gas and star formation rate tracers ([CII]/TIR, $[\mathrm{CII}] / \mathrm{CO}$, and $[\mathrm{CII}] / \mathrm{PAH})$ reveals a pronounced "[CII]-deficit" in the center. In combination with models, a radially declining trend of alpha_CO is found, reflecting the radially declining metallicity gradient in this galaxy. Finally, the overall low alpha_CO values argue for a surprisingly low dark molecular gas content in this galaxy, which is in contrast to estimates in the Milky Way.

FIFI-LS has also studied the [CII] emission in relation to other SFR tracers in multiple other galaxies. One example are the observations of the central region of the active galaxy NGC 4258 by Appleton et al. ${ }^{9}$. The results suggest that a significant share of the [CII] emission from the NGC 4258 is not associated with star formation, but is excited by shocks and turbulence induced by the highly inclined jet of the galaxy. Observing with FIFI-LS, Smirnova-Pinchukova et al. ${ }^{10}$ found excessive [CII] emission in an AGN galaxy as a likely signature of an AGN-driven outflow.

Observations of the Galactic Center presented by Iserlohe et al. ${ }^{11}$ show how the ability of FIFI-LS to quickly map large areas in multiple fine diagnostic lines was used to characterize photo dissociation regions (PDR). Line emission maps of four lines: [CII], [OI] $63 \mu \mathrm{m}$ and $145 \mu \mathrm{m}$, and CO J=14-13 as well as the FIR flux derived using FIFI-LS, FORCAST ${ }^{12}$ and PACS continuum data were used to infer the density and FUV-field using PDR models ${ }^{13}$. The analysis in Iserlohe et al. shows that the [OI] line fluxes at $\sim 63 \mu \mathrm{m}$ have to be affected by self-absorption, while there is significant contribution to the [CII] line flux not originating from the probed PDR. The ability to measure the [OI] line at $145 \mu \mathrm{m}$ and the CO line in addition to [CII] and [OI] at $63 \mu \mathrm{m}$, were the key to derive the correct PDR parameters.

\section{FIFI+LS: THE NEXT LEVEL}

\subsection{Concept overview \& estimated performance improvements}

The sensitivity, FOV and instantaneous spectral coverage of FIFI-LS are currently limited by its 90s-era photoconductor arrays. All of these performance parameters can be improved significantly by upgrading the instrument with new KID 
arrays. These arrays may be produced with an order of magnitude more pixels, enabling the increase of the FOV and the instantaneous spectral coverage of the instrument. KIDs have less generation-recombination (g-r) noise than photoconductors, which translates into a $\sqrt{2}$ sensitivity gain for background-limited operation.

The presented instrument upgrade concept, called FIFI+LS, will maximize the science output while keeping cost and development time low by utilizing most of the original FIFI-LS design and components. This is possible because the original instrument design anticipated future detector upgrades and the entrance optics were designed to accommodate a larger FOV. Table 2 shows the baseline parameters for the FIFI+LS upgrade in comparison to the current FIFI-LS instrument.

Table 2. Upgraded FIFI+LS instrument parameters compared to FIFI-LS

\begin{tabular}{ccccc} 
& $\begin{array}{c}\text { FIFI+LS } \\
\text { Blue Chan. }\end{array}$ & $\begin{array}{c}\text { FIFI+LS } \\
\text { Red Chan. }\end{array}$ & $\begin{array}{c}\text { FIFI-LS } \\
\text { Blue Chan. }\end{array}$ & $\begin{array}{c}\text { FIFI-LS } \\
\text { Red Chan. }\end{array}$ \\
\hline Wavelength Range $[\mu \mathrm{m}]$ & $51-125$ & $115-206$ & $51-125$ & $115-203$ \\
Spect. Res. [km/s] & $130-435$ & $160-425$ & $155-550$ & $160-425$ \\
Instant Spect. Range [km/s] & $2500-9000$ & $3200-10000$ & $800-3000$ & $800-2550$ \\
Field-Of-View & $45^{\prime \prime} \times 35^{\prime \prime}$ & $90^{\prime \prime} \times 70^{\prime \prime}$ & $30^{\prime \prime} \times 30^{\prime \prime}$ & $60^{\prime \prime} \times 60^{\prime \prime}$ \\
Spatial Pixels & $9 \times 7$ & $9 \times 7$ & $5 \times 5$ & $5 \times 5$ \\
Spatial Pixel Pitch & $5^{\prime \prime}$ & $10^{\prime \prime}$ & $6^{\prime \prime}$ & $12^{\prime \prime}$ \\
Spect. Pixels per Spaxel & 64 & 64 & 16 & 16 \\
Image Slices & 7 & 7 & 5 & 5 \\
Detector Width [Pixel]* & $(9+2) \times 7=77$ & $(9+2) \times 7=77$ & $5 \times 5=25$ & $5 \times 5=25$ \\
Detector Size [Pixel $\left.{ }^{2}\right]$ & $77 \times 64=4928$ & $77 \times 64=4928$ & $25 \times 16=400$ & $25 \times 16=400$ \\
\hline
\end{tabular}

* 2 additional pixels per slice to allow for gaps and avoid crosstalk between slices

On the detector level, the instrument's sensitivity will improve by a factor of 1.4 to 2.5 . The lower end of the estimate is the gain from the reduced g-r-noise. Additional gains are expected from improvements of the QE. The current FIFI-LS detectors have a QE of 25-35\% ${ }^{4}$. Depending on the implementation, the new KIDs arrays could yield QEs of 40-80\%. The FOV of the instrument will increase by a factor of 1.75. This increase will enable faster mapping and more efficient observation modes (e.g. Lissajous scanning).

The increased number of spectral pixels will have three distinct advantages. 1. It will facilitate the observation of wide lines, especially in extragalactic sources, providing overall better baseline coverage. 2 . It will enable the observation of atmospheric water features concurrent with the observations for most of the important FIR-diagnostic lines. This will improve the accuracy of the atmospheric calibration and strongly reduce the overhead necessary for atmospheric calibration observations. 3. The detector may be split into two (or more) sections in spectral direction, each optimized for a sub-band of the spectral coverage in the respective channel. This will allow increasing the sensitivity at key lines (e.g. $[\mathrm{CII}]$ and $[\mathrm{OI}])$.

Through a careful redesign of the spectrometer optics, it will be possible to improve the spectral resolution in the blue channel and to increase the spectral range of the red channel to include the [NII] $205 \mu \mathrm{m}$ line. In all, we expect the improvements to increase the observing speed by a factor of 3.5 to 12 for compact sources and for mapping. This gain in efficiency will increase the observable universe for SOFIA/FIFI-LS and provide more efficient use of SOFIA's precious observing time.

The three major areas of the instrument that are affected by the upgrade are the detectors and detector-readout, the IFU and spectrometer optics, and the cooling concept. The necessary changes are addressed in the following sub-sections.

\subsection{KIDS arrays}

There have been important improvements in detector technology since FIFI-LS and PACS fielded the largest germanium gallium-doped photoconductor detector arrays ( 400 pixels), especially in respect to KIDs. The Caltech/JPL group is developing sensitive feedhorn-coupled aluminum KIDs for use at 240-420 $\mu \mathrm{m}$ in the balloon borne spectrometer TIM $^{62}$. TIM will deploy 3600 KIDs using $~ 1,000$ pixel subarrays, and has an NEP requirement of $1 \times 10^{-17} \mathrm{~W} \mathrm{~Hz}^{-1 / 2}$, similar to the photon-limited NEP needed for FIFI+LS. TIM prototype detectors have demonstrated NEPs of $4 \times 10^{-18} \mathrm{~W} \mathrm{~Hz}^{-1 / 2}{ }^{14}$, and simulations indicate an $80 \%$ dual-polarization optical efficiency across the 317-420 micron band ${ }^{15}$. This design will be scaled to the shorter wavelengths of the FIFI+LS red channel. 
The KIDs developed for TIM will also form the basis for the FIFI+LS blue channel detectors, but will use a modified absorber and optical coupling scheme appropriate for the shorter wavelengths. Recent simulation work has shown that thin aluminum film may be patterned on a silicon substrate to produce good optical absorption at wavelengths as short as $10 \mu \mathrm{m}^{16}$. The short meander design studied by Perido et al. ${ }^{16}$ uses a $200 \mathrm{~nm}$ wide absorber line that is interrupted with a meander to increase the resistance per unit length, while simultaneously introducing a distributed capacitance to compensate for the accompanying distributed inductance. For backside illumination, this design achieves a dualpolarization efficiency of 58\% over the 10-30 micron band, a fractional bandwidth larger than that of the 51-125 micron FIFI+LS blue channel spectral range. This absorber geometry will be the starting point of a future optimization targeting the FIFI+LS blue band. The absorber will be illuminated with a microlens equipped with a broadband anti-reflective layer.

The performance of the FIFI+LS KIDs will be similar to those developed for TIM. The diffraction-limited spot size produced by the microlens on the blue channel detectors will be smaller than the wavelength. Assuming a $125 \mu \mathrm{m}$ diameter absorber the inductor volume would be a factor of $\sim 3$ larger than in the TIM prototypes. The capacitor area would be reduced to maintain the LC resonance frequency at $\sim 300 \mathrm{MHz}$, and the expected detector NEP would be $\sim 1.5 \times 10^{-17} \mathrm{~W} \mathrm{~Hz}^{-1 / 2}$. The absorber geometry, pixel size, and layout of the microlens and feedhorn arrays for the blue and red channels has to be refined during a further study. In addition, the detector response within the FIFI+LS spectral bands (especially the large bandwidth of the blue channel) has to be optimized and strategies for maximizing array efficiency at the most used wavelengths have to be investigated, all while retaining background-limited performance. One possible strategy is to split the blue channel detector into two sections along the spectral direction. Those sections would be optimized individually (e.g. considering the polarizing effects of the grating shown in Figure 2).

The switch from background-limited photoconductors to KIDs will improve the point source sensitivity. A photon absorbed in a photoconductor excites a single charge carrier, and the stochastic recombination of these charge carriers increases the fundamental photon noise by a factor of $\sqrt{2}$. In contrast, a photon absorbed in a KID breaks a large number of superconducting electron pairs ( $\sim 17$ for $\lambda=100 \mu \mathrm{m}$ in an aluminum film), and the associated recombination noise is negligible. Additionally, the absorption efficiency in the FIFI+LS KIDs is anticipated to be $\sim 40 \%-80 \%$, an improvement on the $25-35 \%$ achieved with the FIFI-LS photoconductors. As a result of these two terms, we expect the KID arrays will provide at least a factor of $\sqrt{2}$ sensitivity increase based on the g-r noise reduction and an increase up to a factor of 2.5 if we can realize the $80 \% \mathrm{QE}$.

The KIDs arrays will also require a redesign of the readout electronics for the FIFI+LS upgrade. Each KID forms a microwave resonant circuit, with changes to the absorbed optical power driving shifts in the resonant frequency. The readout electronics drive each KID at its resonant frequency, and determine the frequency shifts by monitoring the complex transmission of the drive tone. We will use the same FPGA-based readout originally developed for the BLAST-TNG instrument ${ }^{61}$ and further developed for TIM and other instruments fielding large-format KID arrays ${ }^{17}$.

\subsection{Optics}

The general optical layout of FIFI+LS will remain the same as FIFI-LS. The upgrade will affect the IFU, the collimator optics and the final detector camera optics. The entrance optics can remain unchanged as they were already designed to support a FOV up to $90^{\prime \prime} \mathrm{x} 90$ ". The diffraction gratings will also be reused for the upgrade. The gratings are already the optimal size for the available space and the groove distance and profile are optimal for the FIFI+LS wavelength range. Not including the detectors, the gratings are the most expensive parts of the instrument optics. Retaining the original gratings saves upgrade costs on the order of $\$ 450 \mathrm{k}$.

Each IFU is composed of three sets of mirrors as explained in sub-section 2.1. Each mirror-set contains as many mirrors as there are slices of the FOV. The increase of the FOV from 30"x 30 " to $45^{\prime \prime} \times 35^{\prime \prime}$ in the blue channel and from 60 "x $60^{\prime \prime}$ to $90^{\prime \prime} \times 70^{\prime \prime}$ in the red channel necessitates an increase in spaxel number to sample the FOV sufficiently. The baseline design uses 9x7 spaxels, with 7 slices of 9 spaxels in the IFU. Therefore, the upgraded IFU will need 7 instead of 5 mirrors for each of its three mirror sets. This baseline design of the IFU has already been modeled in the optical software ZEMAX. The model shows that the optics will remain diffraction limited even in the corners of the larger FOV. The additional mirrors will not increase the volume of the complete IFU significantly. This is accomplished by reducing the size of the capture mirrors. Our ZEMAX model has shown that this is possible without affecting the optical performance. The final optimization of the complete spectrometer optics in both channels has to be done in a future study. The currently baselined spaxel pitch of 5" in the blue and 10" in the red channel is a compromise between instantaneous spatial resolution and light losses due to diffraction within the IFU. Within the future study, it has to be determined whether this baseline is the best solution in terms of mapping speed and sensitivity with a full model of the optics. 


\subsection{Cooling}

The new KID arrays operate at temperatures below $250 \mathrm{mK}$, which is considerably lower than the current FIFI-LS detectors' operating temperature of $1.6 \mathrm{~K}$. It will therefore be necessary to redesign the instrument cooling. The original cryostat provides four temperature levels: room temperature, liquid nitrogen cooled at $77 \mathrm{~K}$, liquid helium cooled at $6 \mathrm{~K}$, and pumped liquid helium at $1.6 \mathrm{~K}$. The entrance optics are on the $77 \mathrm{~K}$ level, the spectrometer optics on the $6 \mathrm{~K}$ level and the detectors on the $1.6 \mathrm{~K}$ level.

There are multiple options to realize the necessary detector cooling within the current layout of the FIFI-LS cryostat with different impacts to the overall design. An option with limited impact would only remove the 2.8 -liter superfluid Helium vessel and use the space freed by the vessel and the KIDS detectors, which are smaller in volume compared to the current photoconductor arrays, to accommodate the new detector cooling systems. A more invasive option would require the removal of the 2.8-liter superfluid Helium vessel and the 35-liter Helium vessel. This option uses a pulse tube cryocooler to cool the spectrometer optics and provide the intercept for the detector cooling system. This concept takes advantage of the cryocooler compressors installed on SOFIA, which are used for the cryocooling of upGREAT ${ }^{18}$.

The detector cooling will be realized by an adiabatic demagnetization refrigerator (ADR) to reach temperatures $<250 \mathrm{mK}$. This approach is similar to the approach taken by the SOFIA instruments HAWC $+{ }^{20}$ and HIRMES ${ }^{19}$.

The optimal layout for the cryogenic cooling has to be defined in a future study. For the pulse tube option, it needs to be decided whether a standard commercial two-stage option or a custom single stage option directly coupled to the liquid nitrogen reservoir would be best. The current heat load on the liquid helium cooled level at $6 \mathrm{~K}$ is $1.3 \mathrm{~W}$. TransMIT, the supplier of the upGreat cryocoolers, offers a model that can accommodate $1.17 \mathrm{~W}$ at $4.2 \mathrm{~K}^{21}$ when paired with one of the two compressor systems on SOFIA. We are therefore confident that an optimized design will allow accommodating the heat load from the FIFI+LS system at a temperature below the $6 \mathrm{~K}$ of the current spectrometer optics. This would reduce the instrument internal background in the red channel. For the detector cooling, the two considered options include a dual stage ADR system and a $3 \mathrm{He}$-cooler backed single stage ADR.

\section{SCIENCE OPPORTUNITIES}

Today, SOFIA provides the only FIR observational capability in the world, which means that FIFI-LS has a prime role as an extragalactic workhorse of FIR spectroscopy (due to its wide spectral coverage). However, FIFI-LS' sensitivity and FOV are limited by its detectors, requiring significant time commitments for large sample observing campaigns, making them often difficult or impossible to achieve in a flight series or two. This has limited the science impact of FIFI-LS on SOFIA. The upgraded FIFI+LS with more sensitive and larger detectors, however, will open a new window into FIR studies of the interstellar medium (ISM). With FIFI+LS, it would be possible to create a coherent set of observations of the main FIR fine-structure lines on physical scales that range from a few parsec (e.g. for Small or Large Magellanic Cloud (SMC, LMC) targets) to kilo-parsec (for a wide range of galaxies in the nearby universe) scales, over a significant sample that would be complementary to many of the Herschel surveys. Such a program cannot be practically completed today with FIFI-LS, but it would be routinely possible with FIFI+LS.

The far-infrared fine-structure lines of $\mathrm{C}, \mathrm{N}$, and $\mathrm{O}$ offer a powerful tool to characterize the ISM of nearby and high- $\mathrm{Z}$ galaxies (e.g., Fischer et al. ${ }^{64}$; Cormier et al. ${ }^{22}$; Díaz-Santos et al. ${ }^{23}$; Herrera-Camus et al. ${ }^{24}$ ). These lines arise from both ionized gas and PDRs, and include the [CII] $158 \mu \mathrm{m}$, [OI] 63 and $145 \mu \mathrm{m}$, [NII] 122 and $205 \mu \mathrm{m}$, [OIII] 88 and $52 \mu \mathrm{m}$, and [NIII] $57 \mu \mathrm{m}$ transitions. FIFI+LS will provide a unique opportunity to observe all these lines in the Milky Way and nearby galaxies, while powerful interferometers such as ALMA and NOEMA will be routinely detecting these lines in galaxies in the $1<\mathrm{z}<8$ redshift range (e.g., Capak et al. ${ }^{25}$; Hashimoto et al. ${ }^{26}$ ). Therefore, observations of nearby galaxies with FIFI+LS, where the physical scales probed are much smaller, galaxy samples are larger and ISM properties can be studied in much more detail, are critical as templates to understand and interpret high-z galaxy observations and as an essential link between Milky Way studies and those at high redshift.

PACS made important contributions to our understanding of the FIR line emission in nearby galaxies, ranging from dwarf, low-metallicity systems to the densest, heavily obscured and most energetic starburst nuclei and luminous infrared galaxies (e.g., Madden et al. ${ }^{27}$; Díaz-Santos et al. ${ }^{28}$; González-Alfonso et al..$^{65}$, Herrera-Camus et al. ${ }^{24}$ ). However, PACS has left many key questions unanswered, such as the origin of the FIR line deficit (see below). The only way to address these questions are more comprehensive observations, building on the PACS and FIFI-LS legacy. The superb mapping speed, large FOV, and sensitivity of FIFI+LS opens up entirely new opportunities by making large surveys of nearby and 
intermediate distance galaxies routinely possible. In combination with multi-wavelength ancillary data, SOFIA/FIFI+LS will create its own legacy by entering a new mode of survey style operations, probing the physical properties of the ISM and the origin of the FIR emission lines in an unprecedented range of parameter space. We have outlined a Legacy Science Program (LSP) as part of our NASA Proposal, targeting 43 nearby and intermediate distant galaxies, and 3 star forming regions within the SMC, that cover a wide range of environments and will be a valuable complement to some of Herschel's large surveys (e.g., KINGFISH, SHINING, GOALS, DGS).

This LSP would map the principal fine-structure FIR lines (e.g., [CII], [OI] $63 \mu \mathrm{m}$, [OIII] $88 \mu \mathrm{m}$ ) over a diverse range of galactic environments, including active galactic and starburst nuclei, spiral arm/inter-arm regions, outskirts of galaxies, HII/PDR complexes, and low-metallicity regions. Such a comprehensive view of the ISM would allow to:

- Understand the physical processes in the ISM giving rise to these key lines, as well as the heating/cooling budgets.

- Characterize the structure and interplay between the multiple phases of the ISM, by combining line emission from the neutral/dense (e.g., [CII] and [OI] $63 \mu \mathrm{m}$ ), ionized (e.g., $\mathrm{H \alpha}$, [NII], [OIII]), molecular (e.g., CO, [CI]), and atomic (HI) gas phases.

- Understand how different environments, ranging from regions with moderate star formation to starburst nuclei and AGN, determine the emission of the main FIR lines. These observations will serve as local templates for the rapidly growing number of $z>1$ galaxies detected in these same lines with powerful interferometers such as ALMA and NOEMA.

- Explore the connection between the major coolants of the neutral atomic gas, the [CII] and [OI] $63 \mu \mathrm{m}$ lines, with the heating of the gas, as traced by the FIR continuum emission and the star formation activity. This will provide key insights into the well-known problem of the "[CII]-deficit" and the question of whether [CII] can reliably be used as a star formation tracer across galaxies.

- Determine the role of metallicity in the star formation process in low metallicity environments, such as the Small and Large Magellanic Clouds and other nearby galaxies.

The number and diversity of physical conditions in the regions that shall be mapped for this program would yield a coherent data set, that will be hard to obtain through a number of smaller disconnected programs and allows addressing the following questions:

\section{What is the physics driving the observed "FIR line deficit" in galaxies?}

It is now well established that the fraction of galaxies that have lower FIR line intensities relative to the dust continuum emission increases as a function of FIR luminosity, radiation field strength, star formation efficiency, among other parameters (e.g., Graciá-Carpio et al. ${ }^{29}$; Herrera-Camus et al. ${ }^{24}$; Díaz-Santos et al. ${ }^{23}$ ). This is commonly known as a the "FIR line deficit" problem. In the case of the [CII] line, the relative decrease of the line with respect to the FIR continuum (by up to two orders of magnitude) was first observed in (U)LIRGs with the Infrared Space Observatory (e.g., Malhotra et al ${ }^{30}$; Luhman et al. ${ }^{66}$ ), and later on kiloparsec scale regions in normal, star-forming galaxies (e.g., Croxall et al. ${ }^{31}$; Smith et al..$^{32}$, Bigiel et al. ${ }^{63}$ ) and starbursts ( $\mathrm{D}^{\prime}$ 'az-Santos et al. ${ }^{23}$; Herrera-Camus et al. ${ }^{24}$ ).

The physical reasons behind the line deficit remain an open question, and several explanations have been proposed, including: (1) reduction of the photoelectric heating efficiency due to the charging or destruction of the small dust grains $^{30,33}$, (2) reaching gas densities in PDR and HII regions higher than the critical density of the transitions ${ }^{34}$, (3) HII regions with a high ionization parameter, implying a larger fraction of the non-ionizing stellar UV is absorbed by dust, thus reducing the fraction of UV photons available to heat the neutral gas ${ }^{29,35,34,67}$, (4) a change in the ionization state of the gas due to the harsh AGN powered radiation field ${ }^{36}$, or a combination of high ionization parameter and high FIR extinction ${ }^{64,65}$.

To address the FIR line deficit, we must observe varying spatial scales and probe the dominant physical conditions of the ISM. Ultimately, this requires a multi-wavelength approach where the properties of the different phases of the ISM including metallicity, radiation field strength (FUV and ionized), AGN activity, star formation efficiency and histories are known. The outlined LSP would be very well suited for this purpose, with a wealth of ancillary data available including tracers of the ionized, molecular, and atomic gas phases. This will provide a unique opportunity to finally disentangle the effect of the different physical mechanisms discussed before on setting the line to FIR continuum ratio in galaxies. 


\section{How reliable is the [CII] line emission as a tracer of star formation?}

The [CII] transition is the major coolant for the neutral atomic gas ${ }^{37}$. Therefore, it is expected that if the ISM is in thermal balance, then the [CII] line will measure the total energy input into the gas by star formation activity. Characterizing the relation between the [CII] emission and the star formation rate is particularly relevant for studies of star-forming galaxies at very high-z $(\mathrm{z}>4)$ which are now routinely detected and spatially resolved in [CII] line emission with ALMA (e.g. Capak et al. ${ }^{25}$; Maiolino et al. ${ }^{38}$; Smith et al..$^{39}$ ).

In nearby dwarf and spiral galaxies the observed relation between [CII] and SFR surface density is almost linear, with a scatter of 0.3 dex (e.g., Herrera-Camus et al. ${ }^{7}$; De Looze et al ${ }^{40}$, Bigiel et al. ${ }^{63}$ ). When including the more energetic and obscured environments found in (U)LIRGs, both the slope and the scatter in the [CII]-SFR relation increase. This is a direct consequence of the "[CII]-deficit" discussed above since for (U)LIRGs the bulk of the star formation activity is traced by the FIR emission. Due to the inclusion of galaxies from the optical IFU surveys CALIFA and MANGA, the LSP is designed to probe a parameter space that is currently unexplored: galaxies and regions within galaxies with SFR surface densities in the $\sim 0.01-1 \mathrm{M} \odot \mathrm{yr}^{-1} \mathrm{kpc}^{-2}$ range. This is right in between the parameter space covered by Herschel PACS observations of kilo-parsec scale regions in nearby galaxies (e.g., from KINGFISH) and (U)LIRGs (e.g., GOALS and SHINING).

This would allow studying the nature and reliability of the [CII]-SFR relationship in the context of full optical spectroscopic information, including the exploration of dependences with metallicity, star-formation history, AGN activity, among others.

What is the metal abundance in HII regions when measured using temperature and extinction insensitive FIR line diagnostics?

Understanding the life-cycle of metals as galaxies evolve is crucial to constrain models of chemical enrichment and explain the tightness of the mass-metallicity relation observed in galaxies up to $\mathrm{z} \sim 3$ (e.g., Tremonti et al. ${ }^{41}$; Zahid et al. ${ }^{42}$ ). One important limitation in this type of study is the large uncertainty $(\sim 1$ dex $)$ associated with gas phase metallicity measurements due to the strong dependence on electron temperature of the optical line diagnostics (e.g., Kewley and Ellison ${ }^{43}$ ). One new and direct alternative to measure gas phase metal abundances is based on the far-infrared [OIII] lines at 52 and $88 \mu \mathrm{m}$. The advantage is that these lines arise from low-lying states - which are temperature-insensitive and nearly unaffected by dust extinction, and can be observed at very high-z with ALMA (e.g., Carniani et al. ${ }^{44}$; Hashimoto et al. $\left.{ }^{26}\right)$.

Combining the [OIII] optical $(5007 \AA)$ and FIR $(88 \mu \mathrm{m})$ transitions, Croxall et al. ${ }^{31}$ successfully applied this method to measure gas phase metallicities in 7 HII regions in the nearby galaxy NGC 628. They find that the derived abundances lie right in between the two commonly used abundance scales that differ with respect to each other by a factor of $\sim 4-$ the empirical model ${ }^{45}$ and the photoionization model ${ }^{46}$. A part of the LSP would target the [OIII] $88 \mu \mathrm{m}$ line in a large sample of HII regions in nearby galaxies that have existing deep and spatially resolved IFU optical data. This would enable the development of a new empirical method based on the FIR [OIII] lines to measure metal abundances that are temperature insensitive and extinction free, and that can be applied to very high-z galaxies.

What is the thermal pressure in the Cold Neutral Medium (CNM)? And how is it connected to the star formation process?

Dynamic and thermal processes regulate the structure of the multi-phase ISM and ultimately establish how galaxies evolve through star formation (e.g., Blitz and Rosolowsky ${ }^{47}$ ). In this context, measuring the thermal pressure in the diffuse, neutral gas is of great interest to constrain models of the structure of the ISM (e.g., WNM/CNM balance; Wolfire et al..$^{37}$ ) and the self-regulation of star formation on kilo-parsec scales (Ostriker et al. ${ }^{48}$; Kim et al. ${ }^{49}$ ). In the Galactic plane, the thermal pressure of the diffuse, neutral gas is found to be in the $\mathrm{P}_{\mathrm{th}} \sim 4500-6800 \mathrm{~K} \mathrm{~cm}^{-3}$ range based on studies of absorption features of neutral carbon and CO towards nearby stars (Jenkins and Tripp ${ }^{50}$; Goldsmith et al. ${ }^{51}$ ). Unfortunately, these methods are not available to measure $\mathrm{P}_{\mathrm{th}}$ in nearby galaxies.

Currently, the one alternative we have to measure $P_{t h}$ in extragalactic sources was first proposed by Kulkarni and Heiles ${ }^{52}$. The method relies on the [CII] cooling rate per hydrogen nucleus $\left(\Lambda_{[\mathrm{CII}]}\right)$, which in regions where the [CII] line excitation is dominated by collisions with hydrogen atoms yields the thermal pressure of the neutral, diffuse gas. Herrera-Camus et al..$^{53}$ applied this method to atomic-dominated regions in nearby galaxies and finds a median thermal pressure of $\mathrm{P}_{\mathrm{th}} / \mathrm{k}$ $\sim 3600 \mathrm{~K} \mathrm{~cm}^{3}$, consistent with Galactic measurements. Moreover, they find that the thermal pressure is about $25 \%$ the dynamical equilibrium pressure, and that this fraction is constant as a function of star formation surface density, as expected 
from models of self-regulated star-formation on kilo-parsec scales (e.g.,Ostriker et al. ${ }^{48}$ ). Thanks to the significant increase in the sensitivity and mapping speed of FIFI+LS, the LSP would be designed to greatly improve the number statistics and accuracy of $\mathrm{P}_{\mathrm{th}}$ measurements by mapping large regions in the outskirts of galaxies where the gas is atomic-dominated. These measurements will provide key constraints on the multi-phase neutral ISM structure (e.g., CNM/WNM balance), dynamical equilibrium (e.g., $\mathrm{P}_{\mathrm{tot}} / \mathrm{P}_{\mathrm{th}}$ ), and connection between the $\mathrm{CNM}$ atomic gas and star-formation activity.

What is the fraction of molecular gas that is not traced by CO line emission on different physical scales and galactic environments?

Analytic and numerical models suggest that the "CO-faint" $\mathrm{H}_{2}$ resides in the translucent part of clouds, where $\mathrm{A}_{V} \leq 2 \mathrm{mag}$ (e.g., Wolfire et al..$^{54}$; Glover et al. ${ }^{55}$ ). At this extinction $\mathrm{H}_{2}$ - which is strongly self-shielding - remains molecular but most carbon still exists as $\mathrm{C}+\left(\right.$ Tielens and Hollenbach ${ }^{56}$; Wolfire et al. ${ }^{57}$; Kaufman et al. ${ }^{13}$ ). This leads to a deficit of CO, but renders these regions visible to SOFIA in [CII] $158 \mu \mathrm{m}$.

This "CO-faint" phase is expected to be important even at solar metallicities and to dominate the $\mathrm{H}_{2}$ budget at low metallicities. Studies of the Small Magellanic Cloud (1/5 solar metallicity) find that the "CO-faint" phase encompass $70 \%$ to $90 \%$ of all the $\mathrm{H} 2$ (Israel ${ }^{58}$; Leroy et al. ${ }^{59}$; Jameson et al. ${ }^{60}$ ). Given that it dominates the $\mathrm{H}_{2}$ mass at low metallicities, our embryonic understanding of the amount of "CO-faint" $\mathrm{H}_{2}$ and its effects on star formation is one of the largest gaps in our picture of galaxy evolution. FIFI+LS observations of the SMC in regions with deep ALMA observations of the CO line would make a significant contribution to the limited number of " $\mathrm{CO}$-faint" $\mathrm{H}_{2}$ studies in low-metallicity environments.

\section{CONCLUSION}

The SOFIA observatory provides an environment in which instruments can be upgraded to make use of the latest technological developments. We have presented a FIFI-LS upgrade with KIDs arrays that will take advantage of this environment to significantly improve the instrument's efficiency and increase the size of the SOFIA observable universe. The upgrade will provide 3.5-12 times faster observing, while reducing upgrade cost by utilizing the already flying FIFI-LS instrument as its solid basis. The increased observing speed and sensitivity will allow transitioning from single/small sample observations to large sample survey programs, probing a large range of physical conditions in the ISM and star forming regions extragalactic and within the Galaxy.

\section{ACKNOWLEDGEMENTS}

SOFIA, the "Stratospheric Observatory for Infrared Astronomy" is a joint project of the Deutsches Zentrum für Luft- und Raumfahrt e.V. (DLR; German Aerospace Centre, grant: 50OK0901) and the National Aeronautics and Space Administration (NASA). It is funded on behalf of DLR by the Federal Ministry of Economics and Technology based on legislation by the German Parliament, the state of Baden-Württemberg, and the Universität Stuttgart. Scientific operation for Germany is coordinated by the German SOFIA-Institute (DSI) of the Universität Stuttgart, in the USA by the Universities Space Research Association (USRA). The commissioning of FIFI-LS was supported by the Bundesministerium für Wirtschaft und Technologie (Federal Ministry of Economics and Technology of the Federal Republic of Germany) through the DLR Space Administration (grant: 50OK1201).

This work was also submitted to the Journal of Astronomical Telescopes, Instruments, and Systems, as manuscript \#20162AS.

\section{REFERENCES}

[1] Fischer, C., Beckman, S., Bryant, A., Colditz, S., Fumi, F., Geis, N., Hamidouche, M., Henning, T., Hönle, R., Iserlohe, C., Klein, R., Krabbe, A., Looney, L., Poglitsch, A., Raab, W., Rebell, F., Rosenthal, D., Savage, M., Schweitzer, M., Trinh, C., Vacca, W., "FIFI-LS: The Field-Imaging Far-Infrared Line Spectrometer on SOFIA", J. Astronom. Instrum., Vol. 7, No. 04, 1840003 (2018); https://doi.org/10.1142/S2251171718400032

[2] Becklin, E. E., Young, E. T., Savage, M. L., "Stratospheric Observatory for Infrared Astronomy (SOFIA)", Proc. SPIE 9973, Infrared Remote Sensing and Instrumentation XXIV, 99730I (14 September 2016); https://doi.org/10.1117/12.2238788 
[3] Looney, L.W., Raab, W., Poglitsch, A., Geis, N., "Realizing Integral Field Spectroscopy in the Far-Infrared", ApJ, Vol. 597, Issue 1, 628-643 (2003); https://doi.org/10.1086/378225

[4] Rosenthal, D., Beeman, J., Geis, N., Looney, L., Poglitsch, A., Park, W., Raab, W., and Urban, A., "16x25 Ge:Ga detector arrays for FIFI LS", Proc. SPIE 4014, 156-163 (2000); https://doi.org/10.1117/12.389096

[5] Poglitsch, A., Waelkens, C., and Geis, N., "The Photodetector Array Camera \& Spectrometer (PACS) for the Herschel Space Observatory", Proc. SPIE 4850, IR Space Telescopes and Instruments, (5 March 2003); https://doi.org/10.1117/12.461232

[6] Pineda, J. L., Fischer, C., Kapala, M., Stutzki, J., Buchbender, C., Goldsmith, P. F., Ziebart, M., Glover, S. C. O., Klessen, R. S., Koda, J., Kramer, C., Mookerjea, B., Sandstrom, K., Scoville, N, Smith, R., "A SOFIA Survey of [C II] in the Galaxy M51. I. [C II] as a Tracer of Star Formation", ApJL 869 L3O (2018); https://doi.org/10.3847/2041-8213/aaf1ad

[7] Herrera-Camus, R., Bolatto, A. D., Wolfire, M. G., Smith, J. D., Croxall, K. V., Kennicutt, R. C., Calzetti, D., Helou, G., Walter, F., Leroy, A. K., Draine, B., Brandl, B. R., Armus, L., Sandstrom, K. M., Dale, D. A., Aniano, G., Meidt, S. E., Boquien, M., Hunt, L. K., Galametz, M., Tabatabaei, F. S., Murphy, E. J., Appleton, P., Roussel, H., Engelbracht, C. , Beirao, P. , “[C II] $158 \mu \mathrm{m}$ Emission as a Star Formation Tracer”, ApJ , Vol 800, Number 1 (2015); https://doi.org/10.1088/0004-637X/800/1/1

[8] Kohno, K., Tosaki, T., Matsushita, S., Vila.VilarÓ, B., Shibatsuka, T., Kawabe, R., "Diffuse and Gravitationally Stable Molecular Gas in the Post-Starburst Galaxy NGC 5195", PASJ, Volume 54, Issue 4, $541-553$ (2002); https://doi.org/10.1093/pasj/54.4.541

[9] Appleton, P. N., Diaz-Santos, T., Fadda, D., Ogle, P., Togi, A., Lanz, L., Alatalo, K., Fischer, C., Rich, J., Guillard, P., "Jet-related Excitation of the [CII] Emission in the Active Galaxy NGC4258 with SOFIA", ApJ, Volume 869, Number 1 (2018); https://doi.org/10.3847/1538-4357/aaed2a

[10] Smirnova-Pinchukova, I., Husemann, B., Busch, G., Appleton, P., Bethermin, M., Combes, F., Croom, S., Davis, T. A., Fischer, C., Gaspari, M., Groves, B., Klein, R., O’Dea, C. P., Pérez-Torres, M., Scharwächter, J., Singha, M., Tremblay, G. R., Urrutia, T., "The Close AGN Reference Survey (CARS) - Discovery of a global [CII] $158 \mu \mathrm{m}$ line excess in AGN HE 1353-1917”, A\&A, Volume 626, L3 (2019); https://doi.org/10.1051/0004-6361/201935577

[11] Iserlohe, C., Bryant, A., Krabbe, A., Beckmann, S., Colditz, S., Fischer, C., Fumi, F., Geis, N., Henning, T. K., Hönle, R., Klein, R., Looney, L. W., Poglitsch, A., Raab, W., Rebell, F., Vacca, W. D., "FIFI-LS Observations of the Circumnuclear Ring - Probing the High-density Phase of the PDR", ApJ, Volume 885, Number 2 (2019); https://doi.org/10.3847/1538-4357/ab391f

[12] Herter, T. L., Adams, J. D., Gull, G. E., Schoenwald, J., Keller, L. D., Pirger, B. E., Henderson, C. P., Stacey, G. J., Nikola, T., De Buizer, J. M., Vacca, W. D., Ennico, K., "FORCAST: A Mid-Infrared Camera for SOFIA", J. Astronom. Instrum., Vol. 7, No. 04, 1840005 (2018); https://doi.org/10.1142/S2251171718400056

[13] Kaufman, M. J., Wolfire, M. G., Hollenbach, D. J., Luhman, M. L., "Far-Infrared and Submillimeter Emission from Galactic and Extragalactic Photodissociation Regions", ApJ, Volume 527, Number 2 (1999); https://doi.org/10.1086/308102

[14] Hailey-Dunsheath, S., Barlis, A. C. M., Aguirre, J. E., Bradford, C. M., Redford, J. G., Billings, T. S., LeDuc, H. G., McKenney, C. M., Hollister, M. I., "Development of Aluminum LEKIDs for Balloon-Borne Far-IR Spectroscopy", Journal of Low Temperature Physics, Volume 193, Issue 5-6, 968-975 (2018); 10.1007/s10909-018-1927-y

[15] Nie, R., Janssen, R. M. J., Bradford, C. M., Filippini, J. P., Hailey-Dunsheath, S., "Optimization of a Quasi-Mesh Absorber for the Terahertz Intensity Mapper," in IEEE Transactions on Terahertz Science and Technology, vol. 10, no. 6, pp. 704-712, 2020; https://doi.org/10.1109/TTHZ.2020.3022020

[16] Perido, J., Glenn, J., Day, P., Fyhrie, A., Leduc, H., Zmuidzinas, J., McKenney, C., "Extending KIDs to the Mid-IR for Future Space and Suborbital Observatories", J Low Temp Phys 199, 696-703, 2020; https://doi.org/10.1007/s10909-020-02364-y

[17] Wilson, G. W., Ade, P., Aretxaga, I., Austermann, J. E., Bardin, J., Barry, P., Beall, J., Berthoud, M., Braeley, A., Bryan, S. A., Burkott, A., Bussan, J., Castillo, E., Chavez, M., DeNigris, N., Doyle, S., Eiben, M., Ferrusca, D., Fissel, L., Gao, J., Gear, W., Gómez, V., Gordon, S., Groppi, C., Gutermuth, R., Heyer, M., Kuczarski, S., Hosseini, M., Offner, S., Pope, A., Schloerb, F. P., Souccar, K., Tang, Y., Wallace, G., Yun, M. S., Mauskopf, P., Kelso, R., Knapp, J., Lunde, E., Mani, H., Mathewson, J., Scannapieco, E., Underhill, M., Hubmayr, J., Vissers, M., Hughes, D. H., Montoya, I. R., Sanchez, D., Velazquez, M., Ventura, S., Pascale, E., Rowe, S., Tucker, C., Novak, G., McMahon, J., Simon, S., , "The TolTEC project: a millimeter wavelength imaging 490 polarimeter (Conference Presentation)," Proc. SPIE 10708, 107080I, 2018; https://doi.org/10.1117/12.2313347 
[18] Risacher, C., Güsten, R., Stutzki, J., Hübers, H.-W., Aladro, R., Bell, A., Buchbender, C., Büchel, D., Csengeri, T., Duran, C., Graf, U. U., Higgins, R. D., Honingh, C. E., Jacobs, K., Justen, M., Klein, B., Mertens, M., Okada, Y., Parikka, A., Pütz, P., Reyes, N., Richert, H., Ricken, O., Riquelme, D., Rothbart, N., Schneider, N., Simon, R., Wienold, M., Wiesemeyer, H., Ziebart, M., Fusco, P., Ronser, S., Wohler, B., "The upGREAT Dual Frequency Heterodyne Arrays for SOFIA", J. Astronom. Instrum., Vol. 7, No. 04, 1840014 (2018); https://doi.org/10.1142/S2251171718400147

[19] Richards, S. N., Moseley, S. H., Stacey G., Greenhouse, M., Kutyrev, A., Arendt, R., Atanasoff, H., Banks, S., Brekosky, R. P., Brown, A.-D., Bulcha, B., Cazeau, T., Choi, M., Colazo, F., Engler, C., Hadjimichael, T., HaysWehle, J., Henderson, C., Hsieh, W.-T., Huang, J., Jenstrom, I., Kelogg, J., Kimball, M., Kovács, A., Leiter, S., Maher, S., McMurray, R., Melnick, G. J., Mentzell, E., Mikula, V., Miller, T. M., Nagler, P., Nikola, T., Oxborrow, J., Pontoppidan, K. M., Rangwala, N., Rhodes, A., Roberge, A., Rosner, S., Rostem, K., Rustemeyer, N., Sharp, E., Sparr, L., Stevanivic, D., Taraschie, P., Temi, P., Vacca, W. D., de Lorenzo, J. V. H., Wohler, B., Wollack, E. J., Wilk, S., "SOFIA-HIRMES: Looking Forward to High-Resolution Mid-infrarEd Spectrometer", J. Astronom. Instrum., Vol. 7, No. 04, 1840015 (2018); https://doi.org/10.1142/S2251171718400159

[20] Harper, D. A., Runyan, M. C., Dowell, C. D., Wirth, C. J., Amato, M., Ames, T., Amiri, M., Banks, S., Bartels, A., Benford, D., J., Berthoud, M., Buchanan, E., Casey, S., Chapman, N. L., Chuss, D. T., Cook, B., Derro, R., Dotson, J. L., Evans, R., Fixsen, D., Gatley, I., Guerra, J. A., Halpern, M., Hamilton, R. T., Hamlin, L.A., Hansen, C. J., Heimsath, S., Hermida, A., Hilton, G. C., Hirsch, R., Hollister, M. I., Hostetter, C. F., Irwin, K., Jhabvala, C. A., Jhabvala, M., Kastner, J., Kovács, A., Lin, S., Loewenstein, R. F., Looney, L. W., Lopez-Rodriguez, E., Maher, S. F., Michail, J. M., Miller, T. M., Moseley, S. H., Novak, G., Pernic, R. J., Rennick, T., Rhody, H., Sandberg, E., Sandford, D., Santos, F. P., Shafer, R., Sharp, E. H., Shirron, P., Siah, J., Silverberg, R., Sparr, L. M., Spotz, R., Staguhn, J. G., Toorian, A. S., Towey, S., Tuttle, J., Vaillancourt, J., Voellmer, G., Volpert, C. G., Wang, S., Wollack, E. J., "HAWC+, the Far-Infrared Camera and Polarimeter for SOFIA", J. Astronom. Instrum., Vol. 7, No. 04, 1840008 (2018); https://doi.org/10.1142/S2251171718400081

[21] TransMIT Center for Adaptive Cryotechnology and Sensors, https://cryo.transmit.de/en/products/lambda-line-pulsetubes-two-stage-4-k-pulse-tube-cold-heads-gm-type, last accessed April 2020

[22] Cormier, D., Madden, S. C., Lebouteiller, V., Abel, N., Hony, S., Galliano, F., Rémy-Ruyer, A., Biegiel, F., Baes, M., Boselli, A., Chevance, M., Cooray, A., De Looze, I., Doublier, V., Galametz, M., Hughes, T., Karczewski, O. L., Lee, M.-Y., Lu, N., Spinoglio, L., "The Herschel Dwarf Galaxy Survey. I. Properties of the low-metallicity ISM from PACS spectroscopy.”, A\&A, Volume 578, A53 (2015); https://doi.org/10.1051/0004-6361/201425207

[23] Díaz-Santos, T., Armus, L., Charmandaris, V., Stierwalt, S., Stacey, G., Malhotra, S., van der Werf, P. P., Howell, J. H., Privon, G. C., Mazzarella, J. M., Goldsmith, P. F., Murphy, E. J., Barcos-Muñoz, L., Linden, S. T., Inami, H., Larson, K. L., Evans, A. S., Appleton, P., Iwasawa, K., Lord, S., Sanders, D. B., Surace, J. A., "A Herschel/PACS Far-infrared Line Emission Survey of Local Luminous Infrared Galaxies”, ApJ, Vol. 846, Number 1 (2017); https://doi.org/10.3847/1538-4357/aa81d7

[24] Herrera-Camus, R., Sturm, E., Graciá-Cario, J., Lutz, D., Contursi, A., Veilleux, S., Fischer, J., González-Alfonso, E., Poglitsch, A., Tacconi, L., Genzel, R., Maiolino, R., Sternberg, A., Davies, R., Verman, A., "SHINING, A Survey of Far-infrared Lines in Nearby Galaxies. I. Survey Description, Observational Trends, and Line Diagnositcs.”, ApJ, Vol. 861, Number 2 (2018); https://doi.org/10.3847/1538-4357/aac0f6

[25] Capak, P. L., Carilli, C., Jones, G., Casey, C. M., Riechers, D., Sheth, K., Carollo, C. M., Ilbert, O., Karim, A., Lefevre, O., Lilly, S., Scoville, N., Smolcic, V., Yan, L., "Galaxies at redshifts 5 to 6 with systematically low dust content and high [C II] emission", Nature, Vol. 522, 455-458 (2015); https://doi.org/10.1038/nature14500

[26] Hashimoto, T., Inoue, A. K., Mawatari, K., Tamura, Y., Matsuo, H., Furusawa, H., Harikane, Y., Shibuya, T., Knudsen, K. K., Kohno, K., Ono, Y., Zackrisson, E., Okamoto, T., Kashikawa, N., Oesch, P. A., Ouchi, M., Ota, K., Shimizu, I., Taniguchi, Y., Umehata, H., Watson, D., “ “Big Three Dragons”: a z = 7.15 Lyman Break Galaxy Detected in [OIII] $88 \mu \mathrm{m}$, [CII] $158 \mu \mathrm{m}$, and Dust Continuum with ALMA”, PASJ, Vol. 71, Issue 4 (2019); https://doi.org/10.1093/pasj/psz049

[27] Madden, S. C., Rémy-Ruyer, A., Galametz, M., Cormier, D., Lebouteiller, V., Galliano, F., Hony, S., Bendo, G. J., Smith, M. W. L., Pohlen, M., Roussel, H., Sauvage, M., Wu, R., Sturm, E., Poglitsch, A., Contursi, A., Doublier, V., Baes, M., Barlow, M. J., Boselli, A., Boquien, M., Carlson, L. R., Ciesla, L., Cooray, A., Cortese, L., de Looze, I., Irwin, J. A., Isaak, K., Kamenetzky, J., Karczewski, O. L., Lu, N., MacHattie, J. A., O’Halloran, B., Parkin, T. J., Rangwala, N., Schirm, M. R. P., Schulz, B., Spinoglio, L., Vaccari, M., Wilson, C. D., Wozniak, H., "An Overview of the Dwarf Galaxy Survey", PASP, Vol. 125, Number 928 (2013); https://doi.org/10.1086/671138 
[28] Díaz-Santos, T., Charmandaris, V., Armus, L., Stierwalt, S., Haan, S., Mazzarella, J. M., Howell, J. H., Veilleux, S., Murphy, E. J., Petric, A. O., Appleton, P., Evans, A. S., Sanders, D. B., Surace, J. A., "The Spatial Extent of (U)LIRGS in the Mid-infrared. II. Feature Emission", ApJ , Vol. 741, Number 1 (2011); https://doi.org/10.1088/0004$\underline{637 X / 741 / 1 / 32}$

[29] Graciá-Carpio, J., Sturm, E., Hailey-Dunsheath, S., Fischer, J., Contursi, A., Poglitsch, A., Genzel, R., GonzálezAlfonso, E., Sternberg, A., Verma, A., Christopher, N., Davies, R., Feuchtgruber, H., de Jong, J. A., Lutz, D. , Tacconi, L. J., "Far-infrared Line Deficits in Galaxies with Extreme L $\{\mathrm{FIR}\} / \mathrm{M}\{\mathrm{H}\{2\}\}$ Ratios", ApJ , Vol. 728, Number 1; https://doi.org/10.1088/2041-8205/728/1/L7

[30] Malhotra, S., Helou, G., Stacey, G., Hollenbach, D., Lord, S., Beichman, C. A., Dinerstein, H., Hunter, D. A., Lo, K. Y., Lu, N. Y., Rubin, R. H., Silbermann, N., Thronson, Jr., H. A., Werner, M. W., "Infrared Space Observatory Measurements of [C II] Line Variations in Galaxies", ApJ , Vol 491, Number 1 (1997); https://doi.org/10.1086/311044

[31] Croxall, K. V., Smith, J. D., Brandl, B. R., Groves, B. A., Kennicutt, R. C., Kreckel, K., Johnson, B. D., Pellegrini, E., Sandstrom, K. M., Walter, F., Armus, L., Beirão, P., Calzetti, D., Dale, D. A., Galametz, M., Hinz, J. L., Hunt, L. K., Koda, J., Schinnerer, E., "Toward a Removal of Temperature Dependencies from Abundance Determinations: NGC 628", ApJ , Vol. 777, Number 2 (2013); https://doi.org/10.1088/0004-637X/777/2/96

[32] Smith, J. D. T., Croxall, K., Draine, B., De Looze, I., Sandstrom, K., Armus, L., Beirão, P., Bolatto, A., Boquien, M., Brandl, B., Crocker, A., Dale, D. A., Galametz, M., Groves, B., Helou, G., Herrera-Camus, R., Hunt, L., Kennicutt, R., Walter, F., Wolfire, M., "The Spatially Resolved [CII] Cooling Line Deficit in Galaxies", ApJ , Vol. 834, Number 1 (2016); https://doi.org/10.3847/1538-4357/834/1/5

[33] Croxall, K. V., Smith, J. D., Wolfire, M. G., Roussel, H., Sandstrom, K. M., Draine, B. T., Aniano, G., Dale, D. A., Armus, L., Beirão, P., Helou, G., Bolatto, A. D., Appleton, P. N., Brandl, B. R., Calzetti, D., Crocker, A. F., Galametz, M., Groves, B. A., Hao, C.-N., Hunt, L. K., Johnson, B. D., Kennicutt, R. C., Koda, J., Krause, O., Li, Y., Meidt, S. E., Murphy, E. J., Rahman, N., Rix, H.-W., Sauvage, M., Schinnerer, E., Walter, F., Wilson, C. D., "Resolving the Far-IR Line Deficit: Photoelectric Heating and Far-IR Line Cooling in NGC 1097 and NGC 4559”, ApJ , Vol. 747, Number 1 (2012); https://doi.org/10.1088/0004-637X/747/1/81

[34] Herrera-Camus, R., Sturm, E., Graciá-Cario, J., Lutz, D., Contursi, A., Veilleux, S., Fischer, J., González-Alfonso, E., Poglitsch, A., Tacconi, L., Genzel, R., Maiolino, R., Sternberg, A., Davies, R., Verman, A., "SHINING, A Survey of Far Infrared Lines in Nearby Galaxies. II: Line-Deficit Models, AGN impact, [CII]-SFR Scaling Relations, and Mass-Metallicity Relation in (U)LIRGS", Vol. 861, Number 2 (2018); https://doi.org/10.3847/1538-4357/aac0f9

[35] Luhman, M. L., S. Satyapal, S., Fischer, J., Wolfire, M. G., Sturm, E., Dudley, C. C., Lutz, D., Genzel, R., "The [C II] 158 Micron Line Deficit in Ultraluminous Infrared Galaxies Revisited”, ApJ , Vol. 594, Number 2 (2003); https://doi.org/10.1086/376965

[36] Langer, W. D., Pineda, J. L., "[C ii] emission from galactic nuclei in the presence of X-rays”, A\&A, Vol. 580, A5 (2015); https://doi.org/10.1051/0004-6361/201525950

[37] Wolfire, M. G., McKee, C. F., Hollenbach, D., Tielens, A. G. G. M., "Neutral Atomic Phases of the Interstellar Medium in the Galaxy", ApJ ,Vol. 587, Number1, 278-311 (2003); https://doi.org/10.1086/368016

[38] Maiolino, R., Carniani, S., Fontana, A., Vallini, L., Pentericci, L., Ferrara, A., Vanzella, E., Grazian, A., Gallerani, S., Castellano, M., Cristiani, S., Brammer, G., Santini, P., Wagg, J., Williams, R., "The assembly of 'normal' galaxies at z 7 probed by ALMA", MNRAS, Vol. 452, Issue 1, 54-68 (2015); https://doi.org/10.1093/mnras/stv1194

[39] Smit, R., Bouwens R. J., Carniani S., Oesch, P. A., Labbé, I., Illingworth, G. D., van der Werf, P., Bradley, L. D., Gonzalez, V., Hodge, J. A., Holwerda, B. W., Maiolino, R., Zheng, W., "Rotation in [C II]-emitting gas in two galaxies at a redshift of 6.8", Nature, Vol. 553, 178-181; https://doi.org/10.1038/nature24631

[40] De Looze, I., Cormier, D., Lebouteiller, V., Madden, S., Baes, M., Bendo, G. J., Boquien, M., Boselli, A., Clements, D. L., Cortese, L., Cooray, A., Galametz, M., Galliano, F., Graciá-Carpio, J., Isaak, K., Karczewski, O. L., Parkin, T. J., Pellegrini, E. W., Rémy-Ruyer, A., Spinoglio, L., Smith, M. W. L., Sturm, E., "The applicability of far-infrared finestructure lines as star formation rate tracers over wide ranges of metallicities and galaxy types", A\&A, Vol. 568, A62 (2014); https://doi.org/10.1051/0004-6361/201322489

[41] Tremonti, C. A., Heckman, T. M., Kauffmann, G., Brinchmann, J., Charlot, S., White, S. D. M., Seibert, M., Peng, E. W., Schlegel, D. J., Uomoto, A., Fukugita, M., Brinkmann, J., "The Origin of the Mass-Metallicity Relation: Insights from 53,000 Star-forming Galaxies in the Sloan Digital Sky Survey”, ApJ , Vol. 613, Number 2, 898-913 (2004); https://doi.org/10.1086/423264

[42] Zahid, H. J., Kewley, L. J., Bresolin, F., "The Mass-Metallicity and Luminosity-Metallicity Relations from DEEP2 at z 0.8”, ApJ , Vol. 730, Number 2, 137 (2011); https://doi.org/10.1088/0004-637X/730/2/137 
[43] Kewley, L. J., Ellison, S. L., "Metallicity Calibrations and the Mass-Metallicity Relation for Star-forming Galaxies", ApJ , Vol. 681, Number 2, 1183-1204 (2008); https://doi.org/10.1086/587500

[44] Carniani, S., Maiolino, R., Amorin, R., Pentericci, L., Pallottini, A., Ferrara, A., Willott, C. J., Smit, R., Matthee, J., Sobral, D., Santini, P., Castellano, M., De Barros, S., Fontana, A., Grazian, A., Guaita, L., "Kiloparsec-scale gaseous clumps and star formation at z = 5-7", MNRAS, Vol. 478, Issue 1 (2018); https://doi.org/10.1093/mnras/sty1088

[45] Pilyugin, L. S., Thuan, T. X., "Oxygen Abundance Determination in H II Regions: The Strong Line IntensitiesAbundance Calibration Revisited”, ApJ , Vol. 631, Number 1, 231-243 (2005); https://doi.org/10.1086/432408

[46] Kobulnicky, H. A., Kewley, L. J., "Metallicities of $0.3<z<1.0$ Galaxies in the GOODS-North Field", ApJ ,Vol. 617 , Number 1, 240-261 (2004); https://doi.org/10.1086/425299

[47] Blitz, L., Rosolowsky, E., "The Role of Pressure in GMC Formation II: The H2-Pressure Relation”, ApJ , Vol. 650, Number 2, 933-944 (2006); https://doi.org/10.1086/505417

[48] Ostriker, E. C., McKee, C. F., Leroy, A. K., "Regulation of Star Formation Rates in Multiphase Galactic Disks: A Thermal/Dynamical Equilibrium Model”, ApJ , Vol. 721, Number 2, 975-994 (2010); https://doi.org/10.1088/0004$637 X / 721 / 2 / 975$

[49] Kim, J.-G., Kim, W.-T., Ostriker, E. C., Skinner, M. A., "Modeling UV Radiation Feedback from Massive Stars. I. Implementation of Adaptive Ray-tracing Method and Tests", ApJ , Vol. 851, Number 2, 93 (2017); https://doi.org/10.3847/1538-4357/aa9b80

[50] Jenkins, E. B., Tripp, T. M., "The Distribution of Thermal Pressures in the Diffuse, Cold Neutral Medium of Our Galaxy. II. An Expanded Survey of Interstellar C I Finestructure Excitations”, ApJ , Vol. 734, Number 1, 65 (2011); https://doi.org/10.1088/0004-637X/734/1/65

[51] Goldsmith, P. F., Yıldız, U. A., Langer, W. D., Pineda, J. L., "Herschel Galactic Plane Survey of [NII] Fine Structure Emission", ApJ , Vol. 814, Number 2, 133 (2015); https://doi.org/10.1088/0004-637X/814/2/133

[52] Kulkarni, S. R., Heiles, C., "The atomic component", In Hollenbach, D. J. and H. A. Thronson, Jr., eds., "Interstellar Processes", vol. 134, Springer, Dordrecht, 87-122; https://doi.org/10.1007/978-94-009-3861-8_5

[53] Herrera-Camus, R., Bolatto, A., Wolfire, M., Ostriker, E., Draine, B., Leroy, A., Sandstrom, K., Hunt, L., Kennicutt, R., Calzetti, D., Smith, J. D., Croxall, K., Galametz, M., de Looze, I., Dale, D., Crocker, A., Groves, B., "Thermal Pressure in the Cold Neutral Medium of Nearby Galaxies", ApJ , Vol. 835, Number2, 201 (2017); https://doi.org/10.3847/1538-4357/835/2/201

[54] Wolfire, M. G., Hollenbach, D., McKee, C. F., "The Dark Molecular Gas", ApJ , Vol. 716, Number 2, 1191-1207 (2010); https://doi.org/10.1088/0004-637X/716/2/1191

[55] Glover, S. C. O., Federrath, C., Mac Low, M.-M., Klessen, R. S., "Modelling CO formation in the turbulent interstellar medium", MNRAS, Vol. 404, Issue 1, 2-29 (2009); https://doi.org/10.1111/j.1365-2966.2009.15718.x

[56] Tielens, A. G. G. M., Hollenbach, D., "Photodissociation regions. I - Basic model. II - A model for the Orion photodissociation region", ApJ , Vol. 291, 722-754 (1985).

[57] Wolfire, M. G., Hollenbach, D., Tielens, A. G. G. M.,"’The correlation of C II 158 micron and CO (J = 1 - 0) line emission", ApJ , Vol. 344, 770-778 (1989); https://doi.org/10.1086/167841

[58] Israel, F. P., "H 2 and its relation to CO in the LMC and other magellanic irregulargalaxies", A\&A, Vol. 328, 471$482(1997)$

[59] Leroy, A. K., Bolatto, A., Gordon, K., Sandstrom, K., Gratier, P., Rosolowsky, E., Engelbracht, C. W., Mizuno, N., Corbelli, E., Fukui, Y., Kawamura, A., "The CO-to-H2 Conversion Factor from Infrared Dust Emission across the Local Group", ApJ , Vol. 737, Number 1, 12 (2011); https://doi.org/10.1088/0004-637X/737/1/12

[60] Jameson, K. E., Bolatto, A. D., Wolfire, M., Warren, S. R., Herrera-Camus, R., Croxall, K., Pellegrini, E., Smith, J.-D., Rubio, M., Indebetouw, M., Israel, F. P., Meixner, M., Roman-Duval, J., van Loon, J. T., Muller, E., Verdugo, C., Zinnecker, H., Okada Y., "First Results from the Herschel and ALMA Spectroscopic Surveys of the SMC: The Relationship between [C II]-bright Gas and CO-bright Gas at Low Metallicity”, ApJ , Vol. 853, Number 2, 111 (2018); https://doi.org/10.3847/1538-4357/aaa4bb

[61] Gordon, S., Dober, B., Sinclair, A., Rowe, S., Bryan, S., Mauskopf, P., Austermann, J., Devlin, M., Dicker, S., Gao, J., Hilton, G. C., Hubmayr, J., Jones, G., Klein, J., Lourie, N. P., McKenney, C., Nati, F., Soler, J. D., Strader, M., Vissers, M., "An Open Source, FPGA-Based LeKID Readout for BLAST-TNG: Pre-Flight Results", J. Astonom. Instrum., Vol. 5, No. 04, 1641003 (2016); https://doi.org/10.1142/S2251171716410038

[62] Vieira, J., Aguirre, J., Bradford, C. M., Filippini, J., Groppi, C., Marrone, D., Bethermin, M., Chang, T.-C., Devlin, M., Dore, O., Fu, J., Hailey-Dunsheath, S., Holder, G., Keating, G., Keenan, R., Kovetz, E., Lagache, G., Mauskopf, P., Narayanan, D., Popping, G.,, Shirokoff, E., Somerville, R., Trumper, I., Uzgil, B., Zmuizinas, J., "The Terahertz 
Intesity Mapper (TIM): an Imaging Spectrometer for Galaxy Evolution Studies at High-Redshift", $30^{\text {th }}$ International Sysmposium on Space THz Technology, 2019; https://arxiv.org/abs/2009.14340

[63] Bigiel, F., De Looze, I., Krabbe, A., Cormier, D., Barnes, A. T., Fischer, C., Bolatto, A. D., Bryant, A., Colditz, S., Geis, N., Herrera-Camus, R., Iserlohe, C., Klein, R., Leroy, A. K., Linz, H., Looney, L. W., Madden, S. C., Poglitsch, A., Stutzki, J., Vacca, W. D., "SOFIA/FIFI-LS Full-disk [CII] Mapping and CO-dark Molecular Gas Across the Nearby Spiral Galaxy NGC6946", accepted to ApJ, 2020

[64] Fischer, J., Abel, N., González-Alfonso, E., Dudley, C., Satyapal, S., van Hoof, P., "A Far-Infrared Spectral Sequence of Galaxies: Trends and Models", ApJ, Vol. 795, Number 2, 117, 2014; https://doi.org/10.1088/0004$637 X / 795 / 2 / 117$

[65] González-Alfonso, E., Fischer, J., Sturm, E., Graciá-Carpio, J, Veilleux, S., Meléndez- M., Lutz, D., Poglitsch, A., Aalto, S., Falstad, N., Soon, H., Farrah, D., Blasco, A., Henkel, C., Contursi, A., Verman, A, Spaans, M., Smith, H.A., Ashby, M., Hailey-Dunsheath, S., "High-Lying OH Absorption, [C II] Deficits, and Extreme LFIR / MH2 Ratios in Galaxies", ApJ, Vol. 800, Number 1, 69, 2015; https://doi.org/10.1088/0004-637X/800/1/69

[66] Luhman, M., Satyapal, S., Fischer, J., Wolfire, M., Cox, P., Lord, S., Smith, H.A., Stacey, G., Unger, S., "Infrared Space Observatory Measurements of a [C II] 158 Micron Line Deficit in Ultraluminous Infrared Galaxies", ApJL, Vol. 504, Number 1, L11-L15, 2003; https://doi.org/10.1086/311562

[67] Abel, N., Dudley, C., Fischer, J., Satyapal, S., van Hoof, P., "Dust-Bounded Ultralumionous Galaxies: Model Predictions for Infrared Spectroscopic Surveys", ApJ, Vol. 701, Number 2, 1147-1160, 2009; https://doi.org/10.1088/0004-637X/701/2/1147 